\title{
QUASAR FORMATION IN HIERARCHICAL STRUCTURE FORMATION MODELS
}

\author{
M.G. HAEHNELT \\ Institute of Astronomy, Madingley Road, Cambridge CB3 OHA
}

\begin{abstract}
Hierarchical cosmogonies can consistently explain the evolution of the quasar population if quasars are short-lived and supermassive black holes form fast in the newly-formed nuclei of dark-matter haloes. Here we investigate the relevant physical processes and show that such a fast formation is plausible. The angular-momentum and the gas-supply problem for the formation/feeding of a supermassive black hole are strongly alleviated compared to a scenario in which gas is transported to the centre by tidal interaction of ready-assembled galaxies. The baryonic component of the newly-formed nucleus will cool catastrophically and settle into a self-gravitating angular momentum-supported disc of radius $\sim 100 \mathrm{pc}$. Gravitational instabilities and/or supernovae-induced turbulence will transport the gas further to the centre within less than $10^{8} \mathrm{yr}$. In nuclei of very massive dark matter haloes with sufficiently deep potential well to retain the gas against feedback processes from massive stars and supernovae, concentration of a major fraction of the gas component of the nucleus within the central 1 pc and subsequent formation of a black hole seem unavoidable. A coeval short phase of efficient star formation could explain the observed high metallicities of quasars.
\end{abstract}

\section{Introduction}

While the existence of supermassive black holes is still not proven beyond doubt, accretion onto black holes seems still to be the only viable explanation for the most fundamental properties of the observed activity in AGN [1]. The question of the formation of quasars is therefore basically the same as the question how supermassive black holes form in the nuclei of galaxies and is closely related to the problem of the fueling of AGN [2]. Recent investigations of the quasar luminosity function and its evolution with redshift $[3,4]$ indicate that quasars are rather short-lived with a life-time $t_{\mathrm{Q}} \sim 10^{8} \mathrm{yr}$ close to the Salpeter time scale $t_{\text {Salp }}=4 \times \epsilon_{\mathrm{rad}, 0.1} 10^{7} \mathrm{yr}$ (the e-folding time for the mass of a black hole accreting at the Eddington limit and radiating with efficiency $\left.\epsilon_{\text {rad }}\right)$. Haehnelt \& Rees [4] demonstrated that the strong evolution of the quasar population can be consistently explained in hierarchical structure formation models if quasars are short-lived and supermassive black holes form fast in the cores of newly-formed dark-matter haloes.

\section{The formation of a supermassive black hole in the newly-formed nucleus of a dark matter halo}

The formation of the nucleus of a dark-matter halo is a complicated multi-phase process. If a halo forms from a linearly growing density fluctuation in the 'fluid' of collisionless dark matter dominating the dynamics of the universe, the inner most dense parts will decouple first from the Hubble flow and collapse until virial 
equilibrium is reached at a radius

$$
R_{\text {vir }} \sim 550\left(\frac{M_{\text {nuc }}}{10^{9} M_{\odot}}\right)^{1 / 3}\left(\frac{1+z_{\text {form }}}{1+4}\right)^{-1}\left(\frac{f_{\text {bar }}}{0.1}\right)^{-1 / 3} \text { pc. }
$$

$z_{\text {form }}$ is the formation redshift, $f_{\mathrm{bar}}$ is the fraction of baryons in the universe and $M_{\text {nuc }}$ is the baryonic mass of the nucleus (inner 1\%) of a dark matter halo with isothermal density profile.

The collisional baryonic component is heated by shocks and feedback processes of massive stars and supernovae. However, this heating is not sufficient for pressure support; the gas will shrink by a factor $\sim f_{\mathrm{bar}} /(2 \lambda)^{2}$ in radius on the dynamical time scale of the nucleus ( $t_{\text {nuc }} \sim 10^{8} \mathrm{yr}$ ) and settle into a self-gravitating angularmomentum supported structure of radius

$$
R_{\text {disc }} \sim 55\left(\frac{M_{\text {nuc }}}{10^{9} M_{\odot}}\right)^{1 / 3}\left(\frac{1+z_{\text {form }}}{1+4}\right)^{-1}\left(\frac{f_{\text {bar }}}{0.1}\right)^{-4 / 3}\left(\frac{\lambda}{0.05}\right)^{2} \mathrm{pc} .
$$

$\lambda=|E|^{1 / 2} J / G M^{5 / 2}$ is the usual angular momentum parameter, which describes the spin-up of the growing density inhomogeneity by tidal torques. During the collapse of the baryonic component the first stars form and some weak magnetic fields might be built up. Gravitational instabilities will keep the self-gravitating 'disc' thick $(h / r \gtrsim 0.1)$ and the disc will be highly viscous due to gravitational instabilities and/or supernova induced turbulence. Magnetic fields could also contribute to a high viscosity, if the disc became strongly magnetized. The matter is transported to the centre in a few hundred dynamical times of the disc and the gas in the disc will form stars very efficiently. Together with an IMF biased to massive stars this could explain the high metallicities observed in quasars [5].

Once the gas is concentrated by a factor $\sim 10-100$ in radius star formation will become more and more difficult. At a radius

$$
R_{\text {coll }}=0.8\left(\frac{M_{\text {nuc }}}{10^{9} M_{\odot}}\right)^{3 / 7}\left(\frac{1+z_{\text {form }}}{1+4}\right)^{3 / 7}\left(\frac{l_{*}}{L_{\odot}}\right)^{-4 / 7}\left(\frac{m_{*}}{M_{\odot}}\right)^{6 / 7}\left(\frac{h / R}{0.1}\right)^{-2 / 7} \mathrm{pc}(3)
$$

the collisional time scale becomes smaller than the Kelvin-Helmholtz time scale $t_{\mathrm{KH}}$ of a contracting protostar of mass $m_{*}$, radius $r_{*}$ and luminosity $l_{*}$ and protostars will be destroyed. This assumes that a fraction $t_{\mathrm{KH}}\left(r_{*}\right) / t_{\text {nuc }}$ of the gas is in the form of protostars of radius $r_{*}$ and that the halo has an isothermal density profile.

Star formation will certainly cease if the energy liberated in collisions of stars exceeds the Eddington luminosity of the gas cloud. This occurs at

$$
R_{\text {edd }}=0.07\left(\frac{\xi}{0.05}\right)^{2 / 9}\left(\frac{M_{\text {nuc }}}{10^{9} M_{\odot}}\right)^{5 / 9}\left(\frac{r_{*}}{R_{\odot}}\right)^{4 / 9}\left(\frac{m_{*}}{M_{\odot}}\right)^{-2 / 9}\left(\frac{h / R}{0.1}\right)^{-2 / 9} \mathrm{pc}
$$

[6], where $\xi$ is the fraction of the mass typically liberated in a stellar collision.

Once the gas has been concentrated so far the formation of a black hole is unavoidable. The exact path of the formation is unclear and several intermediate 


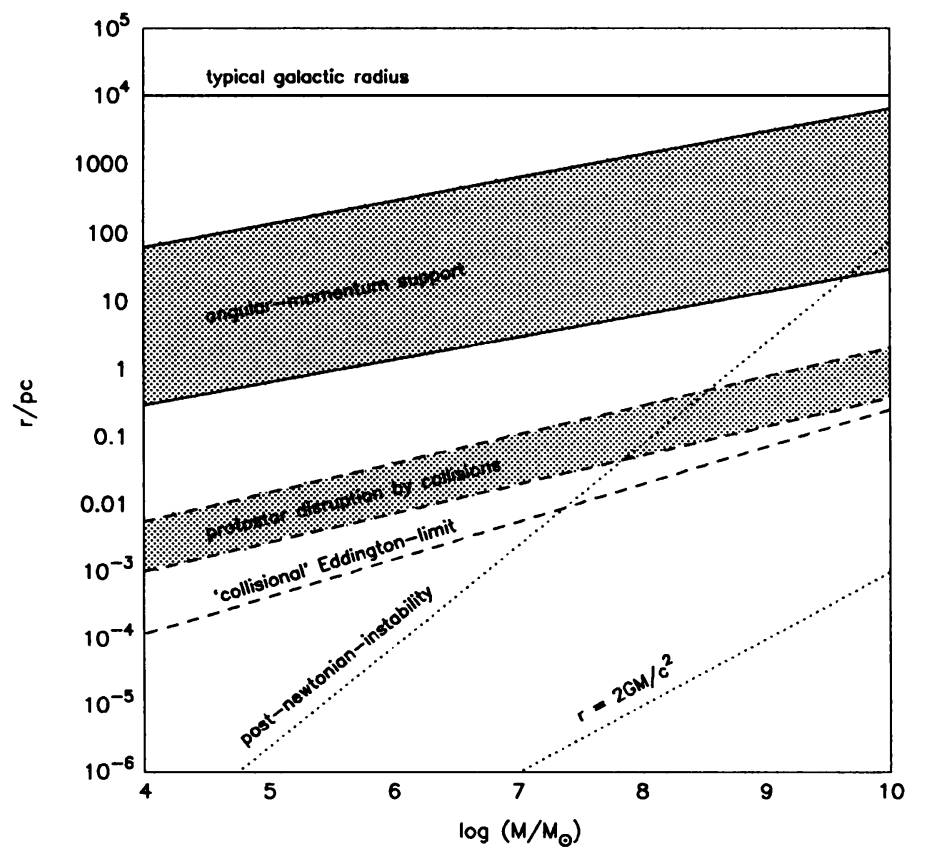

Fig. 1. Characteristic radii for the formation of a supermassive black hole in the newly-formed nucleus of a dark-matter halo as a function of baryonic mass of the nucleus. The upper shaded area shows the range of radii where angular-momentum support of the gas sets in and the lower shaded area shows the range of radii where disruption of protostars by collisions would inhibit star formation. The highest solid line and the middle dashed line are for a halo of constant density, a small formation redshift $z=1$ (and the canonical value of the angular momentum parameter $\lambda=\mathbf{0 . 0 5}$ for the upper shaded area). The opposite limits of the shaded regions are for a halo with isothermal density profile, a high redshift $z=4$ (and a small $\lambda=0.025$ ). The lowest dashed line shows the radius where the luminosity produced by collisional disruption of stars equals the Eddington luminosity of the gas cloud. The dotted lines indicate the Schwarzschild radius and the radius where the post-newtonian instability of a supermassive star sets in.

stages might be involved. However, all of them would be extremely short-lived compared to the dynamical time of the nucleus in which the object is formed [7]. One possible path would be the formation of a supermassive star, which would be unstable for a radius smaller than

$$
R_{\mathrm{pni}}=2.4\left(\frac{M_{\mathrm{sms}}}{10^{9} M_{\odot}}\right)^{3 / 2} \mathrm{pc}
$$

due to a post-newtonian instability. 


\section{Discussion}

Figure 1 shows the different characteristic radii introduced in the last section. It is easily seen how much the angular-momentum problem is alleviated compared to the situation where gas in a ready-assembled galaxy has to be transported inward from radii of order $10 \mathrm{kpc}$. In summary, the transport of the gas to the centre is so much easier in a newly-forming nucleus for the following reasons:

- A self-gravitating disc undergoing violent star formation is certainly highly viscous . Furthermore angular-momentum is not necessarily conserved in the time-varying, non-axisymmetric potential of the newly-forming nucleus.

- The ratio of the radius where angular-momentum support sets in and the radius where the gas can neither form stars nor settle into a stable gaseous configuration can be as small as $\sim 10-100$. A comparatively moderate loss of angular momentum is therefore required.

- The dynamical timescale of the angular-momentum supported disc is short and many dynamical times are available for the inward transport of the matter.

A supermassive black hole can therefore form fast in the newly-formed nucleus (within $t_{\text {nuc }}$ ). The only way to avoid the formation of a black hole would be to turn all the gas of the self-gravitating disc into stars before a significant fraction has reached the centre. For early-forming, very massive, low- $\lambda$ haloes this would require an abnormally high star formation efficiency. In general the black hole formation efficiency should strongly depend on the redshift of formation as the gap between the radius where angular-momentum support sets in and the radius where star formation becomes impossible decreases with increasing redshift for a halo of given mass $\left(R_{\text {disc }} / R_{\text {coll }} \propto(1+z)^{-10 / 7}\right)$. A second important parameter is the depth of the potential well which determines the ability to retain the gas against feedback processes like winds of massive stars and supernovae.

\section{Acknowledgements}

I would like to thank Martin Rees for helpful discussions and acknowledge support by the Gottlieb Daimler- and Karl Benz-Foundation.

\section{References}

1. Blandford R.D., Rees M.J., 1992, in Holt S.W., ed., Testing the AGN paradigm. American Institute of Physics, New York, p1

2. Shlosman I., Begelman M.C., Frank J., 1990, Nat, 345, 679

3. Haehnelt M.G., 1993, in Akerlof C.W., Srednicki M.A., ed., Annals of the New York Academy of Sciences Vol. 688, New York, p. 526

4. Haehnelt M.G., Rees M.J., 1993, MNRAS, 263, 168

5. Hamann F., Ferland G., 1992, ApJ, 391, L53

6. Begelman M.C., Rees M.J., 1978, MNRAS, 185, 847

7. Rees M.J., 1984, ARA\&A, 22, 471 